\title{
Flow and oxygen transport in lung airways
}

\author{
Malcolm Roderick Davidson
}

This thesis is a theoretical study of the transport of gases in lung airway models. Particular emphasis is placed on the transport of oxygen during air breathing and on convection and diffusion of gases in the respiratory region.

Quasi-steady creeping flow in various models of small airway units of the lung is first investigated. A respiratory unit of the lung is modelled by a sphere, an oblate and a prolate ellipsoid of revolution, and a circular cylinder of finite length. The solution of the Stokes equations for each of these geometries is indicated for general axi-symmetric boundary conditions. For particular cases consistent with the models, streamlines are plotted and some velocity profiles are shown. It is suggested that bulk flow in the final generations of the lung is significant for gas transport even though diffusion is the predominant mechanism there.

Next, a pathway through the system of branching in the respiratory region of the lung is modelled by a circular cylinder, closed at one end, with partitions which define the component respiratory units. In this model the transport of oxygen during inspiration, generated by diffusion, is compared with that produced by diffusion together with convection, and the importance of convection in the respiratory region in promoting oxygen uptake at the alveolar wall is discussed. The equations are solved numerically, using the hopscotch method, for different flow rates representing light, moderate, and heavy breathing. It is concluded that

Received 21 February 1974. Thesis submitted to the University of Queensland, March 1973. Degree approved, February 1974. Supervisor: Dr J.M. Fitz-Gerald. 
convection has a significant influence on gas transport in the respiratory region during moderate breathing and that it assumes an increasingly important role as the flow rate increases.

Using similar techniques, the thesis next investigates the concentration of oxygen in expired gas from a model airway system following an inspiration of air during both light and moderate breathing.

Ventilation and perfusion are assumed to be homogeneous in the lung model and the influence of gas exchange on lung gas concentrations is ignored. It is concluded that for moderate air breathing, the experimental alveolar slope reflects either regional inhomogeneities in the lung, or continuing respiratory gas exchange, or (more probably) a combination of both and that, for quiet breathing, the slope also reflects a slow approach to equilibrium of gas concentrations particularly in the early part of the plateau.

Finally, the general problem of gas transport with unequal exchange of $\mathrm{O}_{2}$ and $\mathrm{CO}_{2}$ at the membrane is discussed. Calculations for $\mathrm{O}_{2}$ are made for a complete cycle of air breathing at a flow rate of. $1201 / \mathrm{min}$ on the assumption of equal exchange rates and a sloping alveolar plateau is obtained. 